\title{
Equivalent Elastic Modulus of Asymmetrical Honeycomb
}

\author{
Dai-Heng Chen and Kenichi Masuda \\ Department of Mechanical Engineering, Tokyo University of Science, Kagurazaka 1-3, Shinjuku-ku, Tokyo 162-8601, Japan
}

Correspondence should be addressed to Kenichi Masuda, masudak@rs.kagu.tus.ac.jp

Received 19 March 2011; Accepted 9 April 2011

Academic Editors: J. Botsis, A. Tounsi, and X. Yang

Copyright () 2011 D.-H. Chen and K. Masuda. This is an open access article distributed under the Creative Commons Attribution License, which permits unrestricted use, distribution, and reproduction in any medium, provided the original work is properly cited.

\begin{abstract}
The equivalent elastic moduli of asymmetrical hexagonal honeycomb are studied by using a theoretical approach. The deformation of honeycomb consists of two types of deformations. The first is deformation inside the unit, which is caused by bending, stretching, and shearing of cell walls and rigid rotation of the unit; the second is relative displacement between units. The equivalent elastic modulus related to a direction parallel to one cell wall of the honeycomb is determined from the relative deformation between units. In addition, a method for calculating other elastic moduli by coordinate transformation is described, and the elastic moduli for various shapes of hexagon, which are obtained by systematically altering the regular hexagon, are investigated. It is found that the maximum compliance $\left.C_{y y}\right|_{\max }$ and the minimum compliance $\left.C_{y y}\right|_{\min }$ of elastic modulus $C_{y y}$ in one rotation of the $(x, y)$ coordinate system vary as the shape of the hexagon is changed. However, $\left.C_{y y}\right|_{\max }$ takes a minimum and $\left.C_{y y}\right|_{\min }$ takes a maximum when the honeycomb cell is a regular hexagon, for which the equivalent elastic moduli are unrelated to the selected coordinate system, and are constant with $C_{11}=C_{22}$.
\end{abstract}

\section{Introduction}

To date, honeycomb materials consisting of regular hexagonal cells or symmetrical hexagonal cells [1-4] have been the subject of extensive research. In the present study, a general method is proposed for finding the equivalent elastic moduli for the two-dimensional (2D) problem of honeycomb consisting of an array of hexagonal cells, including asymmetrical hexagonal cells. Moreover, the equivalent elastic moduli for several hexagonal geometries are found using the proposed method, and a systematic investigation is carried out into the effects of changes in geometry of hexagonal cells on the equivalent elastic moduli of honeycomb.

Research into the equivalent elastic moduli of asymmetrical honeycombs has been carried out by Overaker et al. [5], who proposed a method for finding the equivalent elastic moduli of an asymmetrical honeycomb by fitting an equivalent strain field to satisfy the displacements of each end of cell wall in one unit. In the present analysis, the method of Overaker et al. [5] is used although we simultaneously attempt to find the strain field via a different approach. Specifically, by treating the deformation of the honeycomb as the sum of the deformation of each cell wall in one unit and the relative displacement between each unit, the equivalent elastic modulus of the honeycomb can be found from the relative displacement between units. The relative displacement between units is determined by the condition of the junctions between the cell wall ends of each adjacent unit after the deformation.

As shown in Figure 1, the analyzed model in the present study is honeycomb consisting of hexagonal cells, and the honeycomb core height is denoted by $h$. In order to form a honeycomb by periodically arraying hexagonal cells, two opposing edges of the hexagon must have the same length and be parallel. Here, the length and thickness are $l_{1}, l_{2}$, and $l_{3}$, and $t_{1}, t_{2}$, and $t_{3}$, respectively, as shown in the figure; the internal angles formed by the hexagon edges are $\gamma_{12}, \gamma_{23}$, and $\gamma_{31}$

$$
\gamma_{12}+\gamma_{23}+\gamma_{31}=2 \pi .
$$

The cell wall material is homogeneous and isotropic with an elastic modulus of $E_{s}$ and Poisson's ratio of $v_{s}$. The aim of this analysis is to find the equivalent elastic modulus for the honeycomb plane problem. Specifically, we want to find the equivalent elastic modulus $C_{i j}(i, j=1,2,3)$, which is 


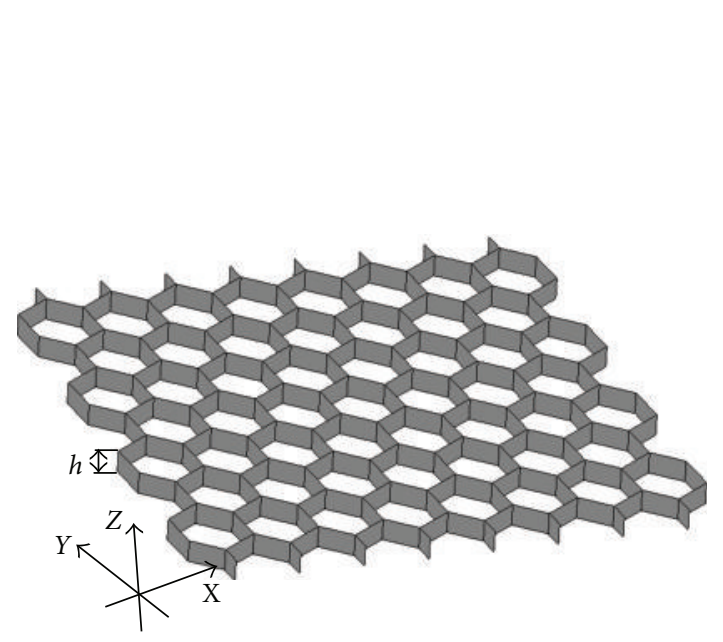

(a)

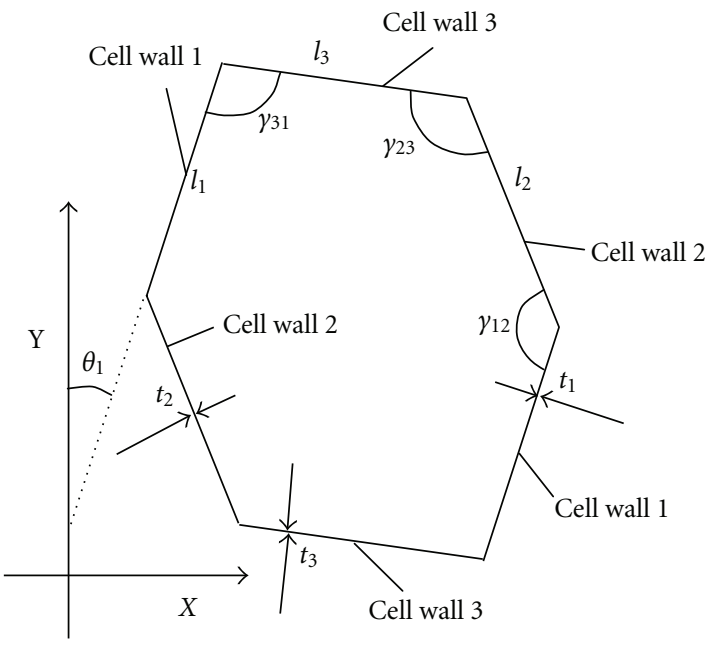

(b)

FIgURE 1: Geometry of honeycomb: (a) honeycomb plate and (b) hexagonal cell.

applicable to the relation of stress and strain in the $x$ - and $y$-coordinate plane shown in Figure 1

$$
\left(\begin{array}{c}
\varepsilon_{x} \\
\varepsilon_{y} \\
\varepsilon_{x y}
\end{array}\right)=\left[\begin{array}{lll}
C_{11} & C_{12} & C_{13} \\
C_{21} & C_{22} & C_{23} \\
C_{31} & C_{32} & C_{33}
\end{array}\right]\left(\begin{array}{c}
\sigma_{x} \\
\sigma_{y} \\
\tau_{x y}
\end{array}\right) .
$$

The shear strain $\varepsilon_{x y}$ used here is defined as a tensor, the engineering definition of which is $\gamma_{x y}$ with $\varepsilon_{x y}=\gamma_{x y} / 2$.

\section{Analysis of Elastic Moduli $C_{12}, C_{22}$, and $C_{32}$ for the $(x, y)$ Coordinates with $\boldsymbol{y}$-Axis Parallel to Edge $\boldsymbol{l}_{1}$}

Initially, the $y$-axis is parallel to cell wall 1 in the case of $\theta_{1}=$ $0^{\circ}$ in Figure 1 , and only the $y$-direction stress $\sigma_{y}$ is considered to act. As shown in Figure 2, the angles $\theta_{2}$ and $\theta_{3}$ are taken as those between edges $l_{2}$ and $l_{1}$, and edges $l_{3}$ and $l_{1}$, respectively

$$
\theta_{2}=\pi-\gamma_{12}, \quad \theta_{3}=\pi-\gamma_{31}
$$

2.1. Force, Moment, and Displacement Acting on Each Cell Wall. Due to the stress $\sigma_{y}$ acting in the $y$-direction, the force $T_{i}(i=1 \sim 3)$ and moment $M_{i}(i=1 \sim 3)$ act on each cell wall, as shown in Figure 3. From the equilibrium of forces, we can obtain

$$
\begin{gathered}
T_{1}+T_{2}=\sigma_{y} h\left(l_{2} \sin \theta_{2}+2 l_{3} \sin \theta_{3}\right), \\
T_{1}=\sigma_{y} h\left(l_{2} \sin \theta_{2}+l_{3} \sin \theta_{3}\right), \\
T_{1}=T_{2}+T_{3},
\end{gathered}
$$



Figure 2: Geometry of hexagon with an edge parallel to $y$-axis $\left(\theta_{1}=0\right)$.

from which, the forces $T_{1}, T_{2}$, and $T_{3}$ are given by the following equations:

$$
\begin{gathered}
T_{1}=\sigma_{y} h\left(l_{3} \sin \theta_{3}+l_{2} \sin \theta_{2}\right), \\
T_{2}=\sigma_{y} h l_{3} \sin \theta_{3}, \\
T_{3}=\sigma_{y} h l_{2} \sin \theta_{2} .
\end{gathered}
$$

The moments due to $T_{i}$ are given as follows:

$$
M_{1}=0 \text {, }
$$

$$
\begin{aligned}
& M_{2}=T_{2} \frac{1}{2} l_{2} \sin \theta_{2}=\frac{1}{2} \sigma_{y} h l_{2} \sin \theta_{2} l_{3} \sin \theta_{3}, \\
& M_{3}=T_{3} \frac{1}{2} l_{3} \sin \theta_{3}=\frac{1}{2} \sigma_{y} h l_{2} \sin \theta_{2} l_{3} \sin \theta_{3} .
\end{aligned}
$$

Here, $M_{2}=M_{3}$. 


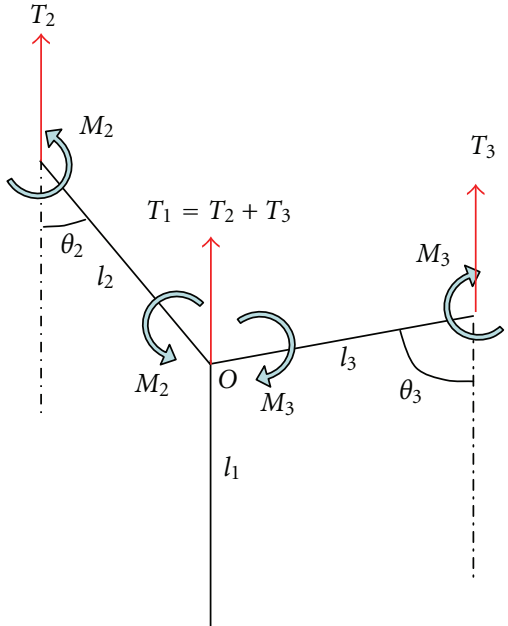

Figure 3: Mechanics of cell walls subjected to stress $\sigma_{y}$ in the $y$ direction.

The $x$-direction displacement $\delta_{i x}$ and $y$-direction displacement $\delta_{i y}$ are found for each cell wall of the unit shown in Figure 4. The bold lines in Figure 4 denote the cell walls, the thin lines denote the boundary of one unit; by arrangement of these units the honeycomb is formed. The displacements of the cell walls are caused by bending deformation, shear deformation and tensile deformation of the cell walls, generated by each force and moment. Taking the junction of the three walls as the origin, the displacements in the $x$-direction of the ends of cell walls 1 , 2 , and 3 are given by the following equations:

$$
\begin{aligned}
\delta_{1 x}= & 0, \\
\delta_{2 x}= & \frac{T_{2} \sin \theta_{2} \cos \theta_{2}}{E_{s} h}\left(\frac{l_{2}}{t_{2}}\right)^{3}+2\left(1+v_{s}\right) \frac{T_{2} l_{2} \sin \theta_{2} \cos \theta_{2}}{k E_{s} h t_{2}} \\
& -\frac{l_{2} T_{2} \cos \theta_{2} \sin \theta_{2}}{E_{s} h t_{2}}, \\
\delta_{3 x}= & -\frac{T_{3} \sin \theta_{3} \cos \theta_{3}}{E_{s} h}\left(\frac{l_{3}}{t_{3}}\right)^{3}-2\left(1+v_{s}\right) \frac{T_{3} l_{3} \sin \theta_{3} \cos \theta_{3}}{k E_{s} h t_{3}} \\
& +\frac{l_{3} T_{3} \cos \theta_{3} \sin \theta_{3}}{E_{s} h t_{3}} .
\end{aligned}
$$

Similarly, the displacements in the $y$-direction of each cell wall are given as follows:

$$
\begin{aligned}
& \delta_{1 y}=-\frac{l_{1} T_{1}}{E_{s} h t_{1}}, \\
& \delta_{2 y}=\frac{T_{2} \sin ^{2} \theta_{2}}{E_{s} h}\left(\frac{l_{2}}{t_{2}}\right)^{3}+2\left(1+v_{s}\right) \frac{T_{2} l_{2} \sin ^{2} \theta_{2}}{k E_{s} h t_{2}}+\frac{l_{2} T_{2} \cos ^{2} \theta_{2}}{E_{s} h t_{2}}, \\
& \delta_{3 y}=\frac{T_{3} \sin ^{2} \theta_{3}}{E_{s} h}\left(\frac{l_{3}}{t_{3}}\right)^{3}+2\left(1+v_{s}\right) \frac{T_{3} l_{3} \sin ^{2} \theta_{3}}{k E_{s} h t_{3}}+\frac{l_{3} T_{3} \cos ^{2} \theta_{3}}{E_{s} h t_{3}} .
\end{aligned}
$$



FIGURE 4: Displacements of ends of cell walls.

Here, the sign of the displacement follows the coordinates shown in Figure 4, and $k$ is a correction coefficient related to shear deformation, which is taken as $k=1$ in this work (previous research has shown that results for $k=1$ agree well with those of numerical analysis by the finite element method [4]).

2.2. Analysis of Equivalent Elastic Moduli. Overaker et al. [5] proposed an elegant method for fixing the equivalent strain field, which satisfies the displacements of each cell wall found in the previous section. Figure 4 shows the displacements of each wall end in a unit $\delta_{i x}$ and $\delta_{i y}$, as well as the coordinates of each wall end $\left(x_{i}, y_{i}\right)(i=1,2,3)$. The displacements in the $x$ - and $y$-directions of each wall end $\delta_{i x}$ and $\delta_{i y}$, which are found in (7) and (8), can be seen as those due to the rigid body displacements $u_{0}$ and $v_{0}$, the rigid body rotation $\omega_{x y}$, and the uniform strain field in the unit $\varepsilon_{x}, \varepsilon_{y}$, and $\varepsilon_{x y}$ and are then described by the following equations:

$$
\begin{aligned}
& \delta_{i x}=x_{i} \varepsilon_{x}+y_{i} \varepsilon_{x y}+y_{i} \omega_{x y}+u_{0}, \\
& \delta_{i y}=y_{i} \varepsilon_{y}+x_{i} \varepsilon_{x y}-x_{i} \omega_{x y}+v_{0} .
\end{aligned} \quad(i=1,2,3)
$$

Six unknowns, namely, $\varepsilon_{x}, \varepsilon_{y}, \varepsilon_{x y}, \omega_{x y}, u_{0}$, and $v_{0}$, are determined by solving these equations. By using these strain fields obtained from (9), the equivalent elastic moduli $C_{12}$, $C_{22}$, and $C_{32}$ can be found from the following equation.

$$
\begin{aligned}
& C_{12}=\frac{\varepsilon_{x}}{\sigma_{y}}, \\
& C_{22}=\frac{\varepsilon_{y}}{\sigma_{y}}, \\
& C_{32}=\frac{\varepsilon_{x y}}{\sigma_{y}} .
\end{aligned}
$$

The strain field produced in the honeycomb can also be determined from the relative displacements between units. In fact, the deformation of the whole honeycomb is performed 
by the relative displacements between each unit. Here, we consider a part of honeycomb consisting of three units, as shown in Figure 5, in which the three units are denoted as units 1, 2, and 3, counterclockwise from the lower left, and the cell wall joints of each unit are $\mathrm{O}_{1}, \mathrm{O}_{2}$, and $\mathrm{O}_{3}$. Denote the relative displacements of $\mathrm{O}_{2}$ and $\mathrm{O}_{3}$ with respect to $\mathrm{O}_{1}$ by $U_{21}$ and $V_{21}$, and $U_{31}$ and $V_{31}$, respectively, as shown in Figure 5(b). Thus, the following equations can be obtained from the relation between the strain field produced in the honeycomb and the displacements of $\mathrm{O}_{2}$ and $\mathrm{O}_{3}$ with respect to $O_{1}$ :

$$
\left(\begin{array}{l}
U_{21} \\
V_{21} \\
U_{31} \\
V_{31}
\end{array}\right)=\left[\begin{array}{cccc}
L_{2 x} & 0 & -L_{2 y} & -L_{2 y} \\
0 & -L_{2 y} & L_{2 x} & -L_{2 x} \\
L_{3 x} & 0 & L_{3 y} & L_{3 y} \\
0 & L_{3 y} & L_{3 x} & -L_{3 x}
\end{array}\right]\left(\begin{array}{c}
\varepsilon_{x} \\
\varepsilon_{y} \\
\varepsilon_{x y} \\
\omega_{x y}
\end{array}\right),
$$

where $L_{2 x}$ and $L_{2 y}$ are the distances in the $x$ - and $y$-directions between $O_{1}$ and $O_{2}$, while $L_{3 x}$ and $L_{3 y}$ are the distances between $\mathrm{O}_{1}$ and $\mathrm{O}_{3}$

$$
\begin{gathered}
L_{2 x}=l_{2} \sin \theta_{2}+l_{3} \sin \theta_{3}, \\
L_{2 y}=l_{2} \cos \theta_{2}-l_{3} \cos \theta_{3}, \\
L_{3 x}=l_{3} \sin \theta_{3}, \\
L_{3 y}=l_{1}+l_{3} \cos \theta_{3} .
\end{gathered}
$$

From (11), the strain field can be obtained as a function of the relative displacements between units as follows:

$$
\begin{gathered}
\varepsilon_{x}=\frac{L_{3 y} U_{21}+L_{2 y} U_{31}}{\left(L_{2 y} L_{3 x}+L_{2 x} L_{3 y}\right)}, \\
\varepsilon_{y}=\frac{-L_{3 x} V_{21}+L_{2 x} V_{31}}{\left(L_{2 y} L_{3 x}+L_{2 x} L_{3 y}\right)}, \\
\varepsilon_{x y}=\frac{-L_{3 x} U_{21}+L_{3 y} V_{21}+L_{2 x} U_{31}+L_{2 y} V_{31}}{2\left(L_{2 y} L_{3 x}+L_{2 x} L_{3 y}\right)}, \\
\omega_{x y}=\frac{-L_{3 x} U_{21}-L_{3 y} V_{21}+L_{2 x} U_{31}-L_{2 y} V_{31}}{2\left(L_{2 y} L_{3 x}+L_{2 x} L_{3 y}\right)} .
\end{gathered}
$$

The relative displacements between units are determined by the condition of the junctions between the wall ends of each adjacent unit. In order to consider the connection between each cell wall after deformation, Figure 5(b) also shows the displacements of points $A, B$, and $C$. Point $A$ of unit 1 is on cell wall 3 , and the displacement of points $A$ with respect to point $O_{1}$ in the respective $x$ - and $y$-direction, $U_{A 1}$ and $V_{A 1}$, are equal to the cell wall deformation itself

$$
\begin{aligned}
& U_{A 1}=\delta_{3 x}, \\
& V_{A 1}=\delta_{3 y} .
\end{aligned}
$$

Point $B$ of unit 2 is on cell wall 2 and point $C$ of unit 3 is on cell wall 1. Therefore, the displacements of points $B$ and $C$ with respect to point $O_{1}$ in the respective $x$ - and $y$-direction,
$U_{i 1}$ and $V_{i 1}(i=B, C)$, are calculated by adding the relative displacements between the units to the displacements due to the cell wall deformation itself

$$
\begin{aligned}
& U_{B 1}=\delta_{2 x}+U_{21}, \\
& V_{B 1}=\delta_{2 y}+V_{21}, \\
& U_{C 1}=\delta_{1 x}+U_{31}, \\
& V_{C 1}=\delta_{1 y}+V_{31} .
\end{aligned}
$$

Since points $A, B$, and $C$ are the same point prior to deformation, as shown in Figure 5(a), the displacement of points $A, B$, and $C$ after deformation must be the same and the condition that $U_{A 1}=U_{B 1}=U_{C 1}$ and $V_{A 1}=V_{B 1}=V_{C 1}$ holds true. From this condition, the relative displacements $U_{21}$ and $V_{21}$, and $U_{31}$ and $V_{31}$ of $O_{2}$ and $O_{3}$ with respect to $\mathrm{O}_{1}$ are given as follows:

$$
\begin{aligned}
& U_{21}=\delta_{3 x}-\delta_{2 x}, \\
& V_{21}=\delta_{3 y}-\delta_{2 y}, \\
& U_{31}=\delta_{3 x}-\delta_{1 x}, \\
& V_{31}=\delta_{3 y}-\delta_{1 y} .
\end{aligned}
$$

By substituting (12) and (16) into (13), the strain field can be obtained, and then, each equivalent elastic modulus $C_{12}, C_{22}$, and $C_{32}$ can be determined from (10).

2.3. Calculation of Elastic Modulus Matrix. In the previous section, we presented a method for finding the three equivalent elastic moduli for the directions parallel to a cell wall constituting the hexagon cell; however, these are only three of the nine components of the elastic modulus described in (2). To express the elastic characteristics of a hexagonal honeycomb, it is necessary to know all nine components. In this section, using the three equivalent elastic moduli relating to the directions parallel to a cell wall, the nine components of the honeycomb equivalent elastic modulus $C_{11}, C_{12} \sim C_{33}$ are derived.

Since the approach described above allows the three equivalent elastic moduli for the direction parallel to any cell wall to be found, the three elastic moduli can be found for each direction of cell walls 1, 2, and 3, respectively. Specifically, as shown in Figure 6, we take the $\left(\alpha^{\prime}, \beta^{\prime}\right)$ coordinates based on cell wall 1 , the $\left(\alpha^{\prime \prime}, \beta^{\prime \prime}\right)$ coordinates based on cell wall 2 and the $\left(\alpha^{\prime \prime \prime}, \beta^{\prime \prime \prime}\right)$ coordinates based on cell wall 3 , in which the $\beta$-axis is set to be parallel to the cell wall. Thus, $C_{12}^{\prime}, C_{22}^{\prime}$, and $C_{32}^{\prime}$ in the $\left(\alpha^{\prime}, \beta^{\prime}\right)$ coordinates, $C_{12}^{\prime \prime}, C_{22}^{\prime \prime}$, and $C_{32}^{\prime \prime}$ in the $\left(\alpha^{\prime \prime}, \beta^{\prime \prime}\right)$ coordinates and $C_{12}^{\prime \prime \prime}, C_{22}^{\prime \prime \prime}$, and $C_{32}^{\prime \prime \prime}$ in the $\left(\alpha^{\prime \prime \prime}, \beta^{\prime \prime \prime}\right)$ coordinates can be found for each coordinate system (the prime superscripts of the coordinate system correspond with those of the elastic moduli). However, the nine components of the elastic modulus $C_{11}, C_{12} \sim C_{33}$ to be found are attached to the $(x, y)$ coordinates of Figure 6 . Thus, we transform coordinates from the $(x, y)$ coordinate system to the $(\alpha, \beta)$ coordinate system. Here, we suppose an angle $\theta$ between the $(x, y)$ 


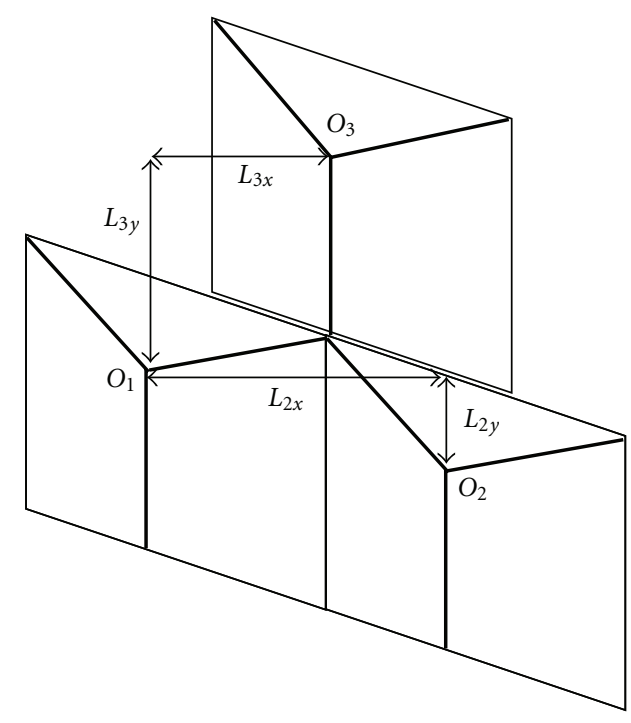

(a)

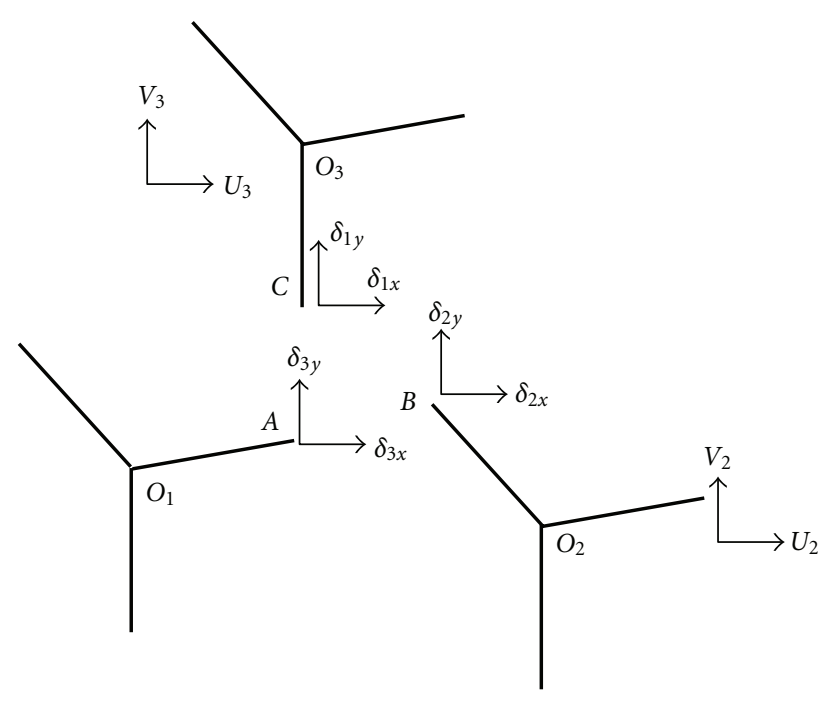

(b)

FIGURE 5: Condition of junctions between wall ends: (a) a part of honeycomb consisting of three units and (b) displacement of cell wall ends $A, B$, and $C$.

coordinate system and the $(\alpha, \beta)$ coordinate system. In the $\left(\alpha^{\prime}, \beta^{\prime}\right)$ coordinate system, $\theta$ is $\theta^{\prime}=\theta_{1}$, for the $\left(\alpha^{\prime \prime}, \beta^{\prime \prime}\right)$ coordinate system, it is $\theta^{\prime \prime}=\theta_{1}+\left(\pi-\gamma_{12}\right)$, and for the $\left(\alpha^{\prime \prime \prime}, \beta^{\prime \prime \prime}\right)$ coordinate system, it is $\theta^{\prime \prime \prime}=\theta_{1}+\left(\pi-\gamma_{12}\right)+(\pi-$ $\left.\gamma_{23}\right)$. For example, by transforming the stress and strain in the $(x, y)$ coordinate system to the stress and strain in the $\left(\alpha^{\prime}, \beta^{\prime}\right)$ coordinate system, the following equation can be obtained from (2):

$$
\left(\begin{array}{c}
\varepsilon_{\alpha^{\prime}} \\
\varepsilon_{\beta^{\prime}} \\
\varepsilon_{\alpha^{\prime} \beta^{\prime}}
\end{array}\right)=[T]^{-1}\left[\begin{array}{lll}
C_{11} & C_{12} & C_{13} \\
C_{21} & C_{22} & C_{23} \\
C_{31} & C_{32} & C_{33}
\end{array}\right][T]\left(\begin{array}{c}
\sigma_{\alpha^{\prime}} \\
\sigma_{\beta^{\prime}} \\
\tau_{\alpha^{\prime} \beta^{\prime}}
\end{array}\right)
$$

The coordinate transformation matrix $[T]$ is given below:

$$
[T]=\left[\begin{array}{ccc}
\cos ^{2} \theta_{1} & \sin ^{2} \theta_{1} & 2 \sin \theta_{1} \cos \theta_{1} \\
\sin ^{2} \theta_{1} & \cos ^{2} \theta_{1} & -2 \sin \theta_{1} \cos \theta_{1} \\
-\sin \theta_{1} \cos \theta_{1} & \sin \theta_{1} \cos \theta_{1} & \cos ^{2} \theta_{1}-\sin ^{2} \theta_{1}
\end{array}\right] .
$$

However, the stress-strain equations in the $\left(\alpha^{\prime}, \beta^{\prime}\right)$ coordinate system are expressed by the following equation:

$$
\left(\begin{array}{c}
\varepsilon_{\alpha^{\prime}} \\
\beta_{\beta^{\prime}} \\
\varepsilon_{\alpha^{\prime} \beta^{\prime}}
\end{array}\right)=\left[\begin{array}{ccc}
C_{11}^{\prime} & C_{12}^{\prime} & C_{13}^{\prime} \\
C_{21}^{\prime} & C_{22}^{\prime} & C_{23}^{\prime} \\
C_{31}^{\prime} & C_{32}^{\prime} & C_{33}^{\prime}
\end{array}\right]\left(\begin{array}{c}
\sigma_{\alpha^{\prime}} \\
\sigma_{\beta^{\prime}} \\
\tau_{\alpha^{\prime} \beta^{\prime}}
\end{array}\right)
$$

Since both (17) and (19) are the same, the following equation is obtained:

$$
\left[\begin{array}{ccc}
C_{11}^{\prime} & C_{12}^{\prime} & C_{13}^{\prime} \\
C_{21}^{\prime} & C_{22}^{\prime} & C_{23}^{\prime} \\
C_{31}^{\prime} & C_{32}^{\prime} & C_{33}^{\prime}
\end{array}\right]=[T]^{-1}\left[\begin{array}{lll}
C_{11} & C_{12} & C_{13} \\
C_{21} & C_{22} & C_{23} \\
C_{31} & C_{32} & C_{33}
\end{array}\right][T] .
$$

As stated above, $C_{12}^{\prime}, C_{22}^{\prime}$, and $C_{32}^{\prime}$ are known and from (20), they can be expressed as functions of the components $C_{11} \sim$ $C_{33}$, which are to be found

$$
\begin{aligned}
C_{12}^{\prime}= & C_{12} \cos ^{4} \theta_{1}-\left(-C_{13}+2 C_{32}\right) \cos ^{3} \theta_{1} \sin \theta_{1} \\
& +\left(C_{11}+C_{22}-2 C_{33}\right) \cos ^{2} \theta_{1} \sin ^{2} \theta_{1} \\
& +\left(C_{23}-2 C_{31}\right) \cos \theta_{1} \sin ^{3} \theta_{1}+C_{21} \sin ^{4} \theta_{1}, \\
C_{22}^{\prime}= & C_{22} \cos ^{4} \theta_{1}+\left(C_{23}+2 C_{32}\right) \cos ^{3} \theta_{1} \sin \theta_{1} \\
& +\left(C_{12}+C_{21}+2 C_{33}\right) \cos ^{2} \theta_{1} \sin ^{2} \theta_{1} \\
& +\left(C_{13}+2 C_{31}\right) \cos \theta_{1} \sin ^{3} \theta_{1}+C_{11} \sin ^{4} \theta_{1}, \\
C_{32}^{\prime}= & C_{32} \cos ^{4} \theta_{1}+\left(C_{12}-C_{22}+C_{33}\right) \cos ^{3} \theta_{1} \sin \theta_{1} \\
& +\left(-C_{23}+C_{31}-C_{32}+C_{13}\right) \cos ^{2} \theta_{1} \sin ^{2} \theta_{1} \\
& +\left(C_{11}-C_{21}-C_{33}\right) \cos \theta_{1} \sin ^{3} \theta_{1}-C_{31} \sin ^{4} \theta_{1} .
\end{aligned}
$$

Similarly, by transforming the $(x, y)$ coordinate system to the $\left(\alpha^{\prime \prime}, \beta^{\prime \prime}\right)$ and $\left(\alpha^{\prime \prime \prime}, \beta^{\prime \prime \prime}\right)$ coordinate systems, $C_{12}^{\prime \prime}, C_{22}^{\prime \prime}$, and $C_{32}^{\prime \prime}$ and $C_{12}^{\prime \prime \prime}, C_{22}^{\prime \prime \prime}$, and $C_{32}^{\prime \prime \prime}$ can be expressed as functions of $C_{11} \sim$ $C_{33}$. Therefore, by solving these nine simultaneous equations, the nine components, $C_{11} \sim C_{33}$, can be determined

By using this method, the honeycomb equivalent elastic components are found. For example, for a cell thickness of 


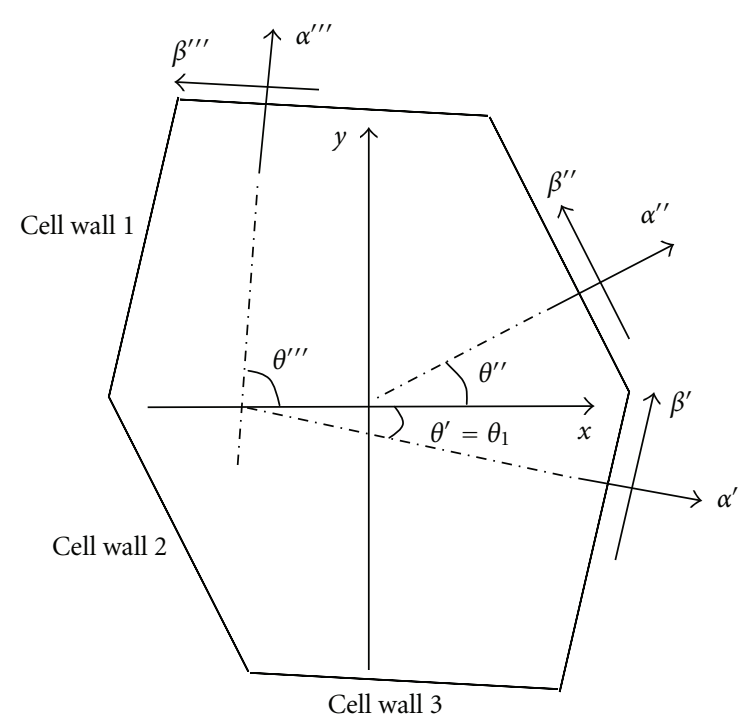

Figure 6: $(\alpha, \beta)$ coordinates based on each cell wall.

$t_{1}=t_{2}=t_{3}=0.05 l_{3}$, Poisson's ratio of $v_{s}=0.3$, the honeycomb equivalent elastic moduli for a hexagon with parameters of $l_{1} / l_{3}=l_{2} / l_{3}=1, \gamma_{12}=140^{\circ}, \gamma_{23}=120^{\circ}$, and $\gamma_{31}=100^{\circ}$ can be determined

$$
[C]=\left[\begin{array}{ccc}
4.076 & -2.458 & 1.505 \\
-2.458 & 2.614 & 3.326 \\
0.753 & 1.663 & 8.568
\end{array}\right] \times \frac{10^{3}}{E_{s}}
$$

Moreover, for a hexagon with parameters of $l_{1} / l_{3}=2.45$, $l_{2} / l_{3}=0.6, \gamma_{12}=153.7^{\circ}, \gamma_{23}=115^{\circ}$, and $\gamma_{31}=91.3^{\circ}$, the following honeycomb equivalent elastic moduli are calculated:

$$
[C]=\left[\begin{array}{ccc}
11.01 & -0.5104 & 20.83 \\
-0.5104 & 0.2750 & 1.709 \\
10.42 & 0.854 & 35.72
\end{array}\right] \times \frac{10^{3}}{E_{s}} .
$$

It can be seen from these results that the symmetry of the elastic moduli holds

$$
\begin{gathered}
C_{21}=C_{12}, \\
2 C_{31}=C_{13}, \\
2 C_{32}=C_{23} .
\end{gathered}
$$

\section{Effects of Geometry on Elastic Moduli}

In order to investigate whether the geometry of hexagonal cell affects each of the equivalent elastic moduli, the equivalent elastic moduli are found for various hexagons that deviates from the regular hexagon, which is taken as a basic geometry here. For the following investigation, in order to observe the effects due to changes in the cell geometry, each cell wall thickness of the basic regular hexagon is taken to be the same, $t_{1}=t_{2}=t_{3}=0.0866 l$. Here, $l$ is the length of one edge of the regular hexagon.
Figure $7(\mathrm{a})$ shows hexagon $A^{\prime} B C D^{\prime} E F$ (geometry 1), which is formed from the regular hexagon $A B C D E F$ by fixing edges $B C$ and $E F$ and moving only points $A$ and $D$ in the $x$ direction by $-\Delta$ and $\Delta$, respectively. Each equivalent elastic modulus corresponding to the hexagonal cell of geometry 1 shown in Figure 7(a) is shown in Figure 8. Here, with the elastic modulus of a regular hexagonal cell $\left.C_{22}\right|_{\text {regular }}$ taken as the standard, the elastic modulus $C_{i j}$ along the vertical axis

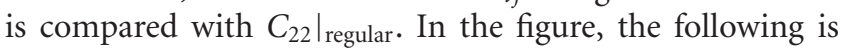
observed.

(1) The elastic modulus $C_{22}$, which expresses the magnitude of the $y$-direction strain due to the stress in the $y$-direction, is a maximum for $\Delta=0$, that is, the regular hexagon, and $C_{22}$ decreases with increasing $\Delta$. When $\Delta /(\sqrt{3} l / 2) \rightarrow 1, C_{22}$ is not 0 but converges to $C_{22} /\left.C_{22}\right|_{\text {regular }}=0.0288$, because hexagon $A^{\prime} B C D^{\prime} E F$ becomes parallelogram $A^{\prime} B D^{\prime} E$ when $\Delta /(\sqrt{3} l / 2)=$ 1 , as shown in Figure $7(\mathrm{~b})$. For the parallelogram $A^{\prime} B D^{\prime} E$, the elastic modulus $C_{22}$ is $C_{22}=\sqrt{3} l /\left(t E_{s}\right)$.

(2) The elastic modulus $C_{11}$, which expresses the magnitude of the $x$-direction strain due to the stress in the $x$-direction, appears not to be strongly influenced by the change in geometry due to the movement of points $A$ and $D$ in the $x$-direction; for each $\Delta, C_{11}$ remains nearly constant.

(3) The elastic modulus $C_{32}$, which expresses the magnitude of the shear strain due to the stress in the $y$ direction, for the case of $\Delta=0$, that is, for the regular hexagon, is zero due to symmetry. As $\Delta$ increases and the geometry deviates from that of a regular hexagon, $\left|C_{32}\right|$ increases; however, in the vicinity of about $\Delta /(\sqrt{3} l / 2) \cong 0.6,\left|C_{32}\right|$ decreases, because shear deformation due to the stress $\sigma_{y}$ decreases, as the geometry approaches that of a parallelogram.

In order to investigate the resultant deformation due to $C_{32}$ and $C_{22}$, we consider the displacement of the upper end $U_{x}$ and $U_{y}$ of a honeycomb plate in geometry 1 under a tensile stress $\sigma_{y}$, as shown in Figure 9(a). For a plate length of $L$ under $\sigma_{y}$, the displacement is given as follows:

$$
U_{x}=\sigma_{y} C_{23} L, \quad U_{y}=\sigma_{y} C_{22} L .
$$

Figure 9 (b) shows the ratio of the compliance $U /\left(\sigma_{y} L\right)$ of the plate to the compliance $\left.C_{22}\right|_{\text {regular }}$ of the regular hexagon. Here, $U=\sqrt{U_{x}^{2}+U_{y}^{2}}$ is the displacement of the upper end of the plate. In Figure 9(b), it can be seen that the comprehensive compliance due to $C_{32}$ and $C_{22}$ increases as $\Delta$ increases and reaches a maximum in the vicinity of $\Delta /(\sqrt{3} l / 2)=0.5$. As $\Delta$ further increases, when geometry 1 deviates from the regular hexagon greatly, the compliance conversely becomes smaller.

(4) The elastic modulus $C_{12}$, which expresses the magnitude of the $x$-direction strain due to stress in the $y$-direction, is always $C_{12}<0$. For $\Delta=0,\left|C_{12}\right|$ is a maximum and becomes zero when $\Delta /(\sqrt{3} l / 2)=1$. 




(a)

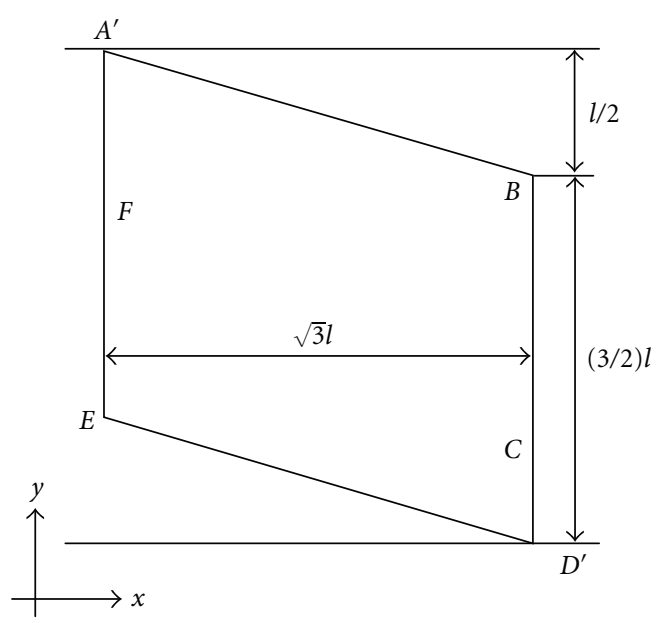

(b)

Figure 7: Geometry 1 of hexagonal cell: (a) horizontal movement of point $A$ and $D$ and (b) parallelogram $(\Delta=\sqrt{3} l / 2)$.

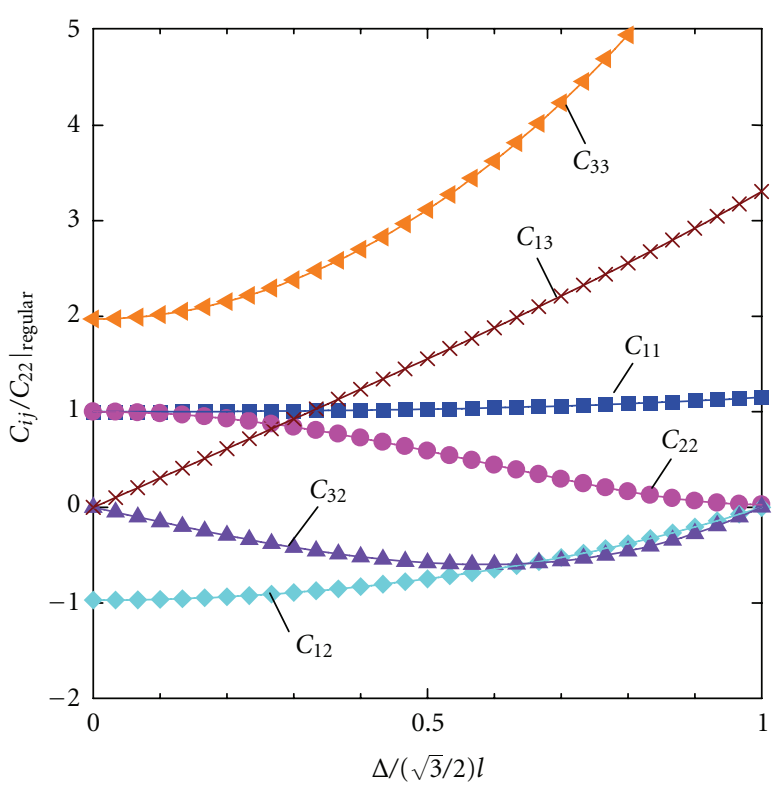

FIGURE 8: Equivalent elastic moduli for geometry 1 shown in Figure 7.

In addition, the ratio of $C_{12}$ and $C_{22}$ is Poisson's ratio $v_{21}$, $v_{21}=-C_{12} / C_{22}$. Figure 10 shows the change in Poisson's ratio $v_{21}$ with changing $\Delta$. Poisson's ratio $v_{21}$ for $\Delta=$ 0 is $v_{21} \cong 0.971$ (as the tensile deformation and the shear deformation of the cell wall are also taken into consideration in the present research, in addition to the bending deformation of the cell wall, $v_{21} \cong 0.971$; however, as indicated by Gibson et al. [3], $v_{21}=1$ when only bending deformation of the cell wall is considered). Near $\Delta /(\sqrt{3} l / 2)=$ 0.9 Poisson's ratio reaches its maximum value of about 3.1.

(5) For geometry 1 of the hexagonal cell, we also investigate the maximum value $\left.C_{y y}\right|_{\max }$ and the minimum value $\left.C_{y y}\right|_{\min }$ of the elastic moduli $C_{y y}$ in one rotation of the $(x, y)$ coordinate axes, which are shown in Figure 11. When $\Delta=0$; that is, when geometry 1 is a regular hexagon, $\left.C_{y y}\right|_{\max }$ is at a minimum, and $\left.C_{y y}\right|_{\min }$ is at a maximum; both equal the elastic modulus of regular hexagonal

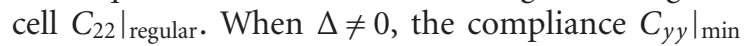
for a certain direction becomes small, however, the compliance $\left.C_{y y}\right|_{\max }$ for other direction becomes large. That is, when deviating the cell form from a regular hexagonal cell, the rigidity of the honeycomb can increase for a certain specific direction; however, direction for which the rigidity becomes small also exists. For the regular hexagon cell, it is found that the equivalent elastic moduli are unrelated to the selected coordinate system, and the compliance of arbitrary direction is always the same as follows:

$$
\begin{gathered}
C_{11}=C_{22}=C_{22} \mid \text { regular } \text { for } y \text {-axis parallel to a cell wall, } \\
C_{12}=C_{12} \mid \text { regular } \quad \text { for } y \text {-axis parallel to a cell wall, } \\
\qquad C_{13}=C_{23}=C_{31}=C_{32}=0 \\
C_{33}=C_{22}-C_{12} .
\end{gathered}
$$

It is not dependent on whether the tensile or the shear deformation is taken into the analysis of equivalent elastic modulus that (26) holds. Equation (26) is based on the characteristic symmetry of the regular hexagon. That is, using the symbols shown in Figure 6, for a regular hexagon, we have

$$
\begin{gathered}
\theta^{\prime}=0, \quad \theta^{\prime \prime}=\pi / 3, \quad \theta^{\prime \prime \prime}=2 \pi / 3, \\
C_{i 2}^{\prime}=C_{i 2}^{\prime \prime}=C_{i 2}^{\prime \prime \prime} \quad(i=1,2,3) .
\end{gathered}
$$




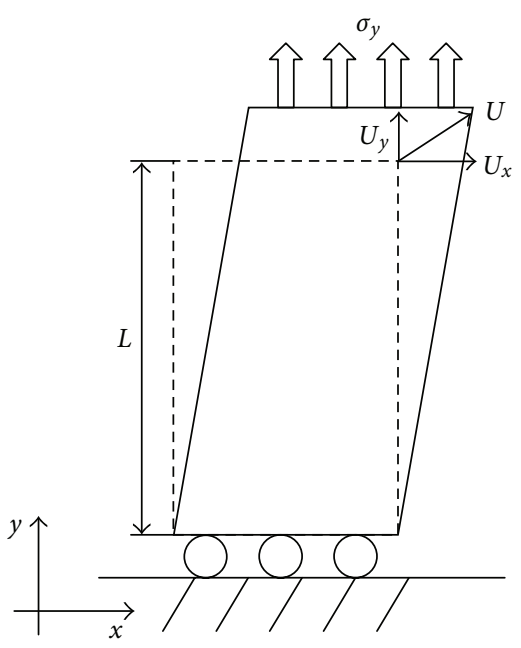

(a)

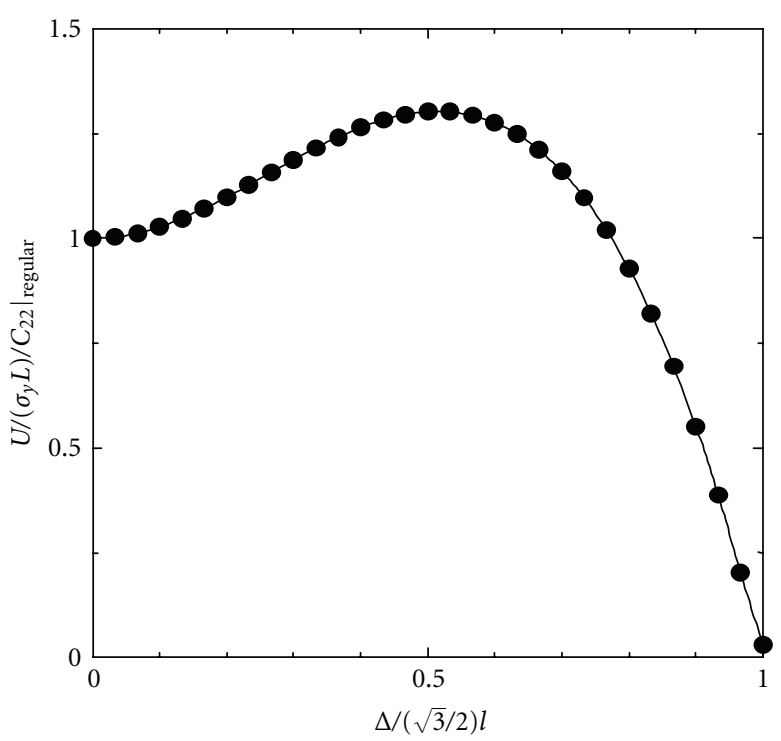

(b)

FIGURE 9: Resultant deformation due to $C_{32}$ and $C_{22}$ : (a) displacement of plate subjected to stress $\sigma_{y}$ and (b) compliance $U /\left(\sigma_{y} L\right)$.

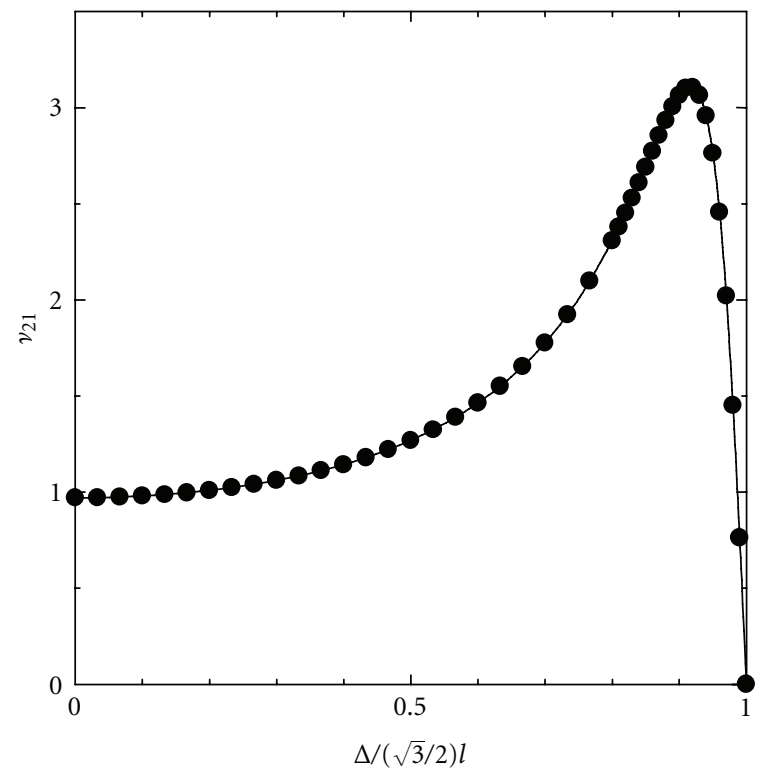

FIGURe 10: Change in Poisson's ratio $v_{21}$ with changing $\Delta$ for geometry 1.

By substituting (27) into (21), (26) can be obtained.

(6) The elastic modulus $C_{33}$, which expresses the magnitude of the shear strain due to the shear stress, is a minimum when $\Delta=0$; however, as the geometry approaches that of a parallelogram, $C_{33}$ becomes larger, since shear deformation is generated easily.

Next, we consider the hexagonal cell $A B^{\prime} C^{\prime} D E^{\prime} F^{\prime}$, which is referred to as geometry 2 here and is formed from the regular hexagon by fixing points $A$ and $D$, and moving points $B, C, E$, and $F$ in the $y$-direction, as shown in

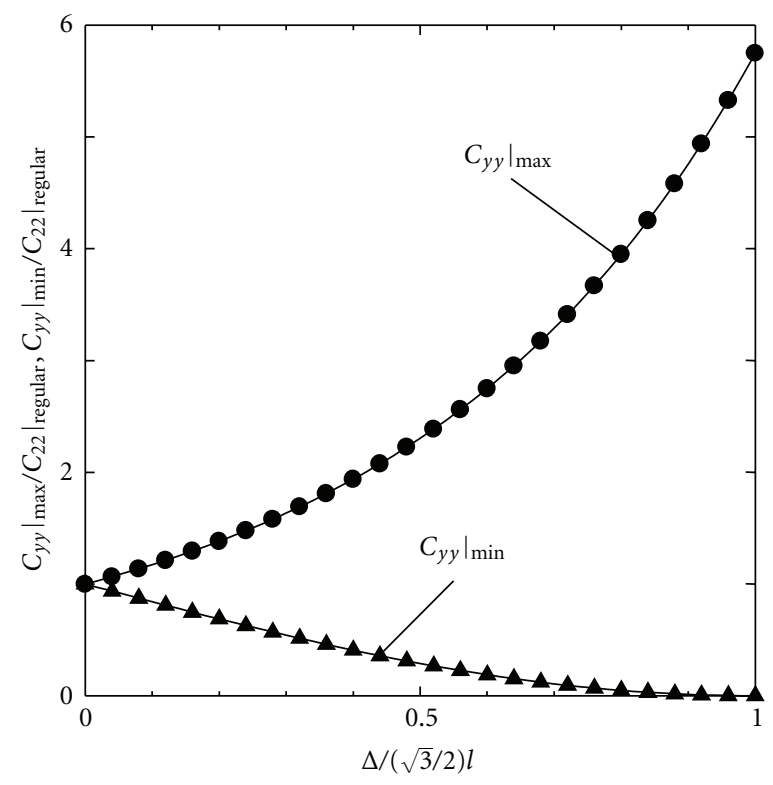

Figure 11: Change in $\left.C_{y y}\right|_{\max }$ and $\left.C_{y y}\right|_{\min }$ with changing $\Delta$ for geometry 1.

Figure 12(a). The nonzero elastic moduli for geometry 2 $\left(C_{32}=C_{13}=0\right.$ from left-right symmetry) are shown in Figure 13. For geometry 2, points $B^{\prime}$ and $C^{\prime}$, as well as points $E^{\prime}$ and $F^{\prime}$, converge when $\Delta=-l / 2$, transforming the hexagon into rhomboid $A B^{\prime} D E^{\prime}$. However, when $\Delta=$ $l / 2$, the three points $A, B^{\prime}$, and $F^{\prime}$ and the three points $C^{\prime}$, $D$, and $E^{\prime}$ form straight lines, transforming the hexagon to rectangle $B^{\prime} C^{\prime} E^{\prime} F^{\prime}$. In Figure 13 , when $\Delta$ changes from the rhomboid to the rectangle, the following is observed. (1) $C_{33}$ becomes large; that is, $C_{33}$ increases from the value of 


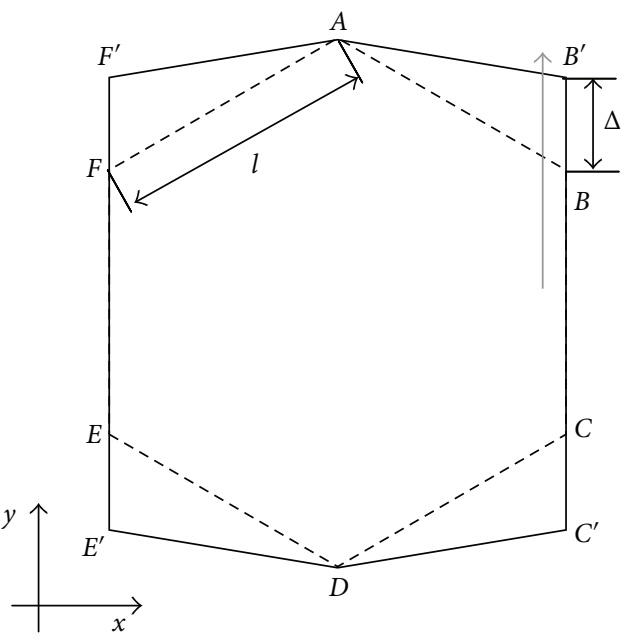

(a)

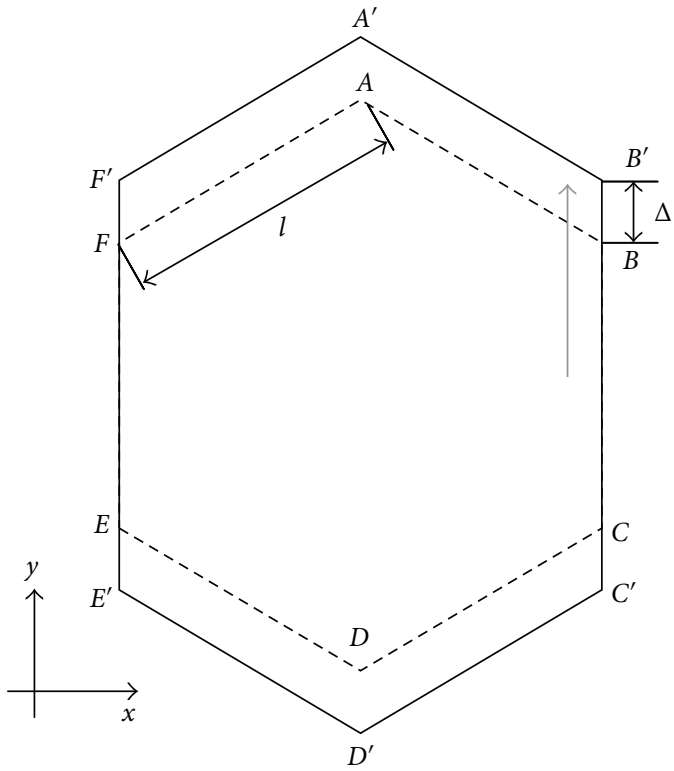

(b)

FiguRE 12: (a) Geometry 2 of hexagonal cell; (b) geometry 3 of hexagonal cell.

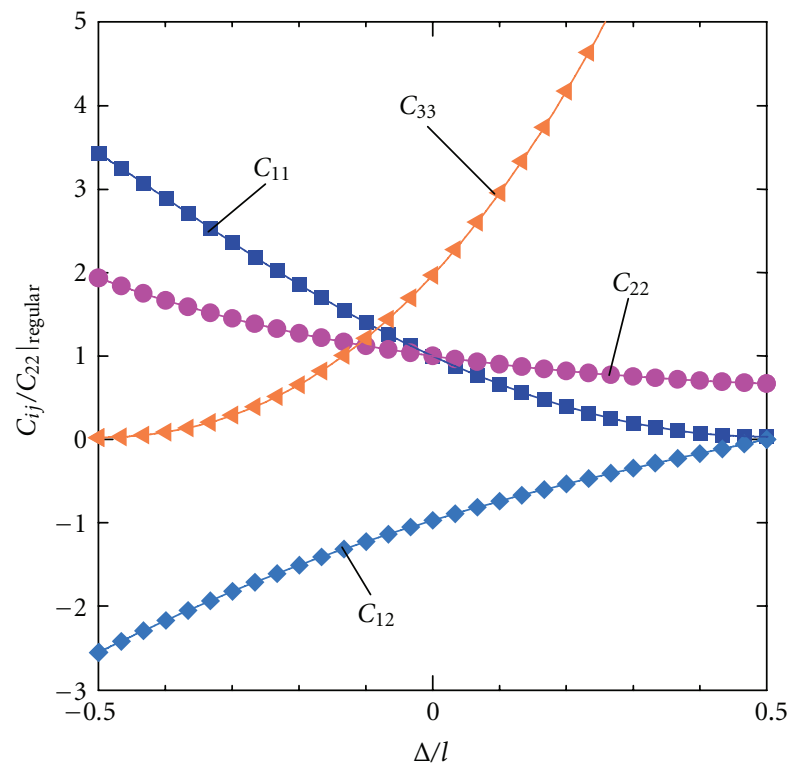

Figure 13: Equivalent elastic moduli for geometry 2 shown in Figure 12(a).

$C_{33} /\left.C_{22}\right|_{\text {regular }}=0.022$ for the rhomboid to $C_{33} /\left.C_{22}\right|_{\text {regular }}=$ 9.42 for the rectangle. (2) The elastic moduli $C_{11}, C_{22}$, and $C_{12}$ (absolute values) each become smaller. Namely, these elastic moduli decrease from the values of $C_{11} /\left.C_{22}\right|_{\text {regular }}=$ $3.43, C_{22} /\left.C_{22}\right|_{\text {regular }}=1.94$ and $C_{12} /\left.C_{22}\right|_{\text {regular }}=-2.56$ for the rhomboid to $C_{11} /\left.C_{22}\right|_{\text {regular }}=0.033, C_{22} /\left.C_{22}\right|_{\text {regular }}=$ 0.67 , and $C_{12}=0$ for the rectangle.

The maximum value $\left.C_{y y}\right|_{\max }$ and the minimum value $\left.C_{y y}\right|_{\min }$ of the elastic modulus $C_{y y}$ in one rotation of the $(x, y)$ coordinate axes are shown in Figure 14. $\left.C_{y y}\right|_{\max }$ and $\left.C_{y y}\right|_{\min }$ take a minimum and a maximum, respectively, when

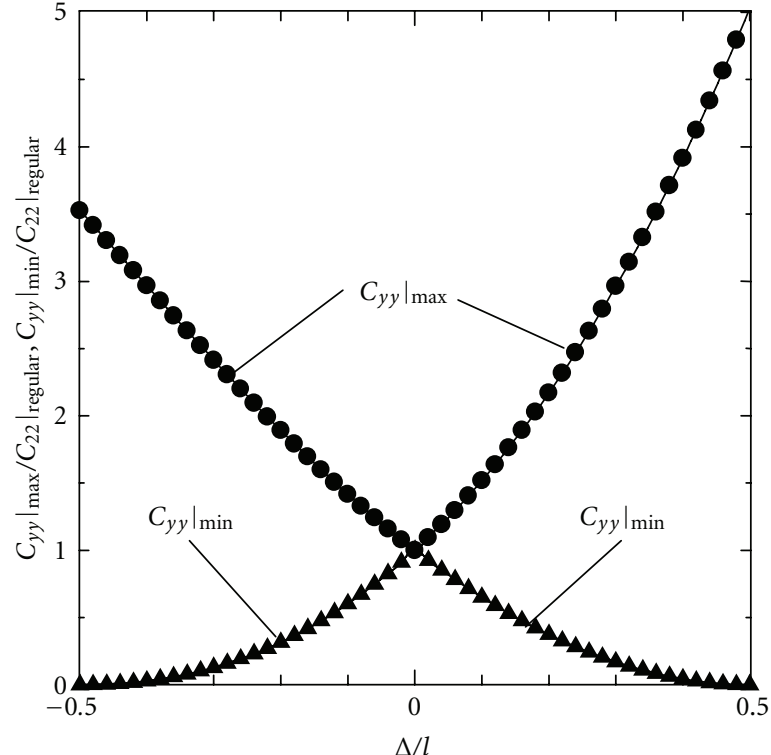

Figure 14: Change in $\left.C_{y y}\right|_{\max }$ and $\left.C_{y y}\right|_{\min }$ with changing $\Delta$ for geometry 2 .

$\Delta=0$, that is, when geometry 2 is a regular hexagon, which is similar to the case of geometry 1 .

Lastly, we consider a hexagonal cell $A^{\prime} B^{\prime} C^{\prime} D^{\prime} E^{\prime} F^{\prime}$, referred to as geometry 3 here, which is formed from the regular hexagon by moving the upper edge $F A B$ and lower edge CDE to the upper and lower sides by $\Delta$ in the $y$-direction, respectively, as shown in Figure 12(b). The nonzero elastic moduli for geometry $3\left(C_{32}=C_{13}=0\right.$ from left-right symmetry) are shown in Figure 15. As shown in the figure, $C_{22}$ decreases. This is because the length of the 


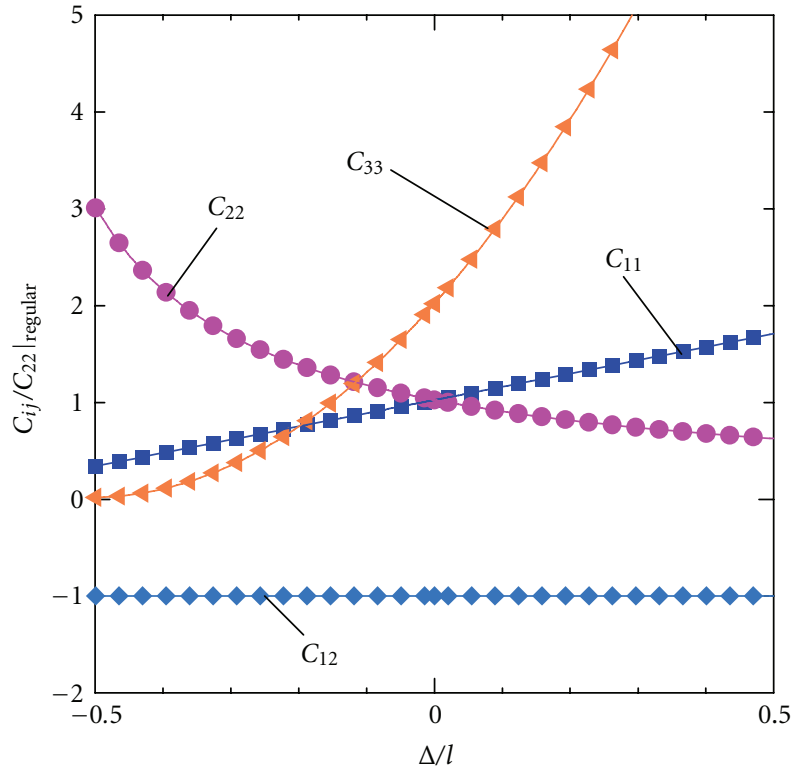

Figure 15: Equivalent elastic moduli for geometry 3 shown in Figure 12(b).



FIgure 16: Change in $\left.C_{y y}\right|_{\max }$ and $\left.C_{y y}\right|_{\min }$ with changing $\Delta$ for geometry 3 .

cell walls parallel to the $y$-axis increases with increasing $\Delta$; these cell walls only undergo tensile deformation, and the amount of deformation is small compared to the bending deformation. Moreover, due to the increase in the length of one unit in the $y$-direction, the force acting at the sloping cell walls due to stress $\sigma_{x}$ increases, thus increasing $C_{11}$. Furthermore, even if $\Delta$ changes, the elastic modulus $C_{12}$ remains constant, maintaining a value of $C_{12} /\left.C_{22}\right|_{\text {regular }}=$ -0.971 . When $\Delta$ changes, the force acting at the sloping cell walls due to the stress $\sigma_{y}$ does not change because the width of one unit does not change in the $x$-direction. Therefore, the deformation of the sloping cell walls is the same and the equivalent strain in the $x$-direction, $\varepsilon_{x}$, also remains the same.

The maximum value $\left.C_{y y}\right|_{\max }$ and the minimum value $\left.C_{y y}\right|_{\min }$ of the elastic modulus $C_{y y}$ in one rotation of the $(x, y)$ coordinate axes are shown in Figure 16, from which it is seen that $\left.C_{y y}\right|_{\max }$ is at a minimum and $\left.C_{y y}\right|_{\min }$ is at a maximum when $\Delta=0$, that is, when geometry 3 is a regular hexagon, as in the cases of geometry 1 and geometry 2 .

\section{Conclusions}

In this research, the equivalent elastic moduli of asymmetrical hexagonal honeycomb are studied by using a theoretical approach. The deformation of honeycomb consists of two types of deformations. The first is deformation inside the unit, which is caused by bending, stretching, and shearing of cell walls and rigid rotation of the unit; the second is relative displacement between units. The relative displacements between units are determined by condition of the junctions between wall ends of each adjacent unit, and the equivalent elastic modulus related to a direction parallel to one cell wall of the honeycomb is determined from the relative deformation between units. In addition, using the three equivalent elastic moduli relating to the directions parallel to the cell wall, the nine components of the honeycomb equivalent elastic modulus $C_{11}, C_{12} \sim C_{33}$ are derived by coordinate transformation. Using the proposed calculation equation, the elastic moduli for various shapes of hexagon, which are obtained by systematically altering the regular hexagon, are investigated. It is found that the maximum compliance $\left.C_{y y}\right|_{\max }$ and the minimum $\left.C_{y y}\right|_{\min }$ of elastic modulus $C_{y y}$ in one rotation of the $(x, y)$ coordinate system vary as the shape of the hexagon is changed. However, $\left.C_{y y}\right|_{\max }$ takes the minimum and $\left.C_{y y}\right|_{\min }$ takes the maximum when the honeycomb cell is a regular hexagon, for which the equivalent elastic moduli are unrelated to the selected coordinate system and are constant with $C_{11}=C_{22}$ and $C_{33}=C_{22}-C_{12}$.

\section{References}

[1] I. G. Masters and K. E. Evans, "Models for the elastic deformation of honeycombs," Composite Structures, vol. 35, no. 4, pp. 403-422, 1996.

[2] W. E. Warren and A. M. Kraynik, "Foam mechanics: the linear elastic response of two-dimensional spatiallly periodic cellular materials," Mechanics of Materials, vol. 6, no. 1, pp. 27-37, 1987.

[3] L. J. Gibson, M. F. Ashby, G. S. Schajer, and C. I. Robertson, "The mechanics of two-dimensional cellular materials," Proceedings of The Royal Society of London, Series A, vol. 382, no. 1782, pp. 25-42, 1982.

[4] D. H. Chen and S. Ozaki, "Analysis of in-plane elastic modulus for a hexagonal honeycomb core: Effect of core height and proposed analytical method," Composite Structures, vol. 88, no. 1, pp. 17-25, 2009.

[5] D. W. Overaker, A. M. Cuitiño, and N. A. Langrana, "Elastoplastic micromechanical modeling of two-dimensional irregular convex and nonconvex (re-entrant) hexagonal foams," Journal of Applied Mechanics, Transactions ASME, vol. 65, no. 3, pp. 748-757, 1998. 

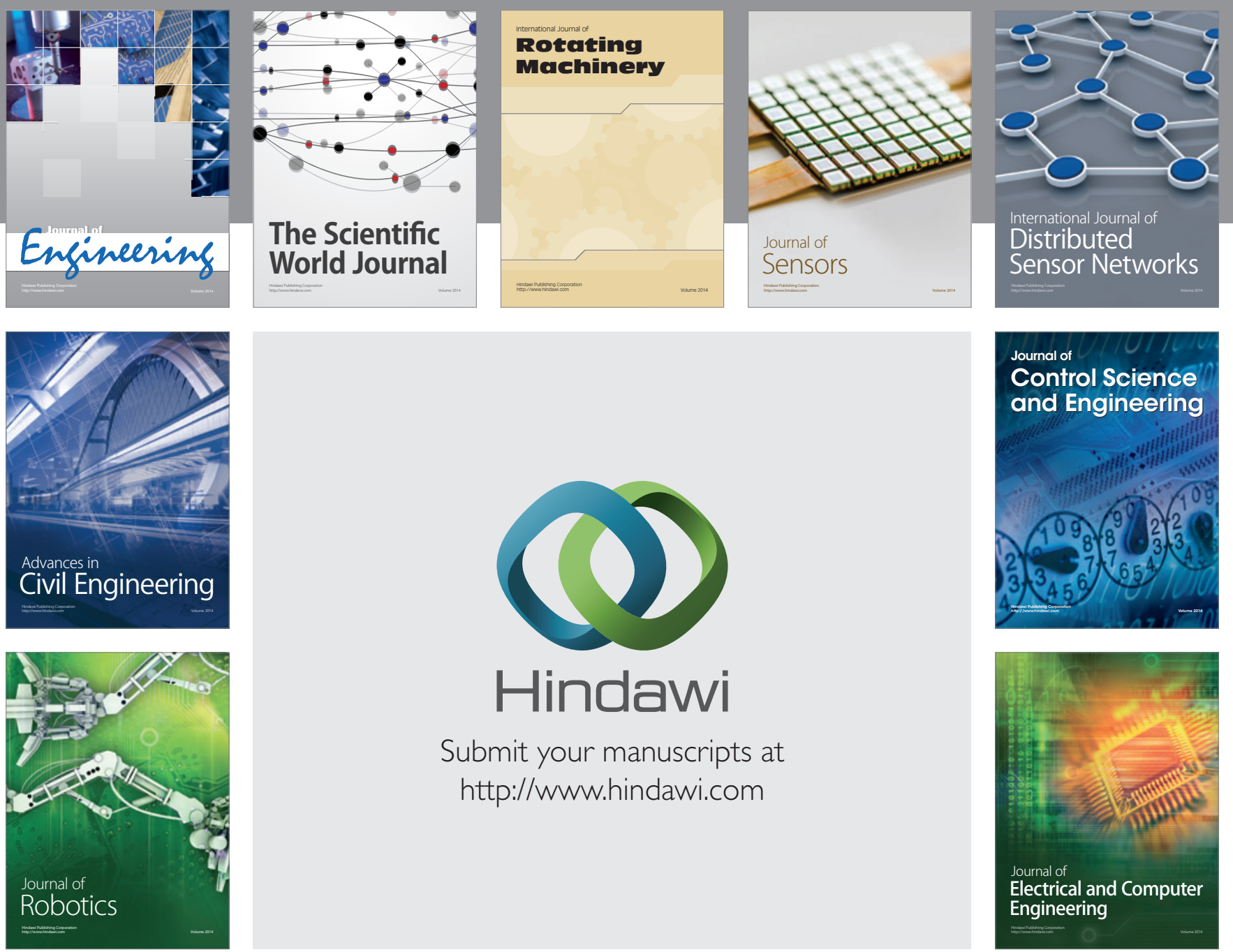

Submit your manuscripts at

http://www.hindawi.com
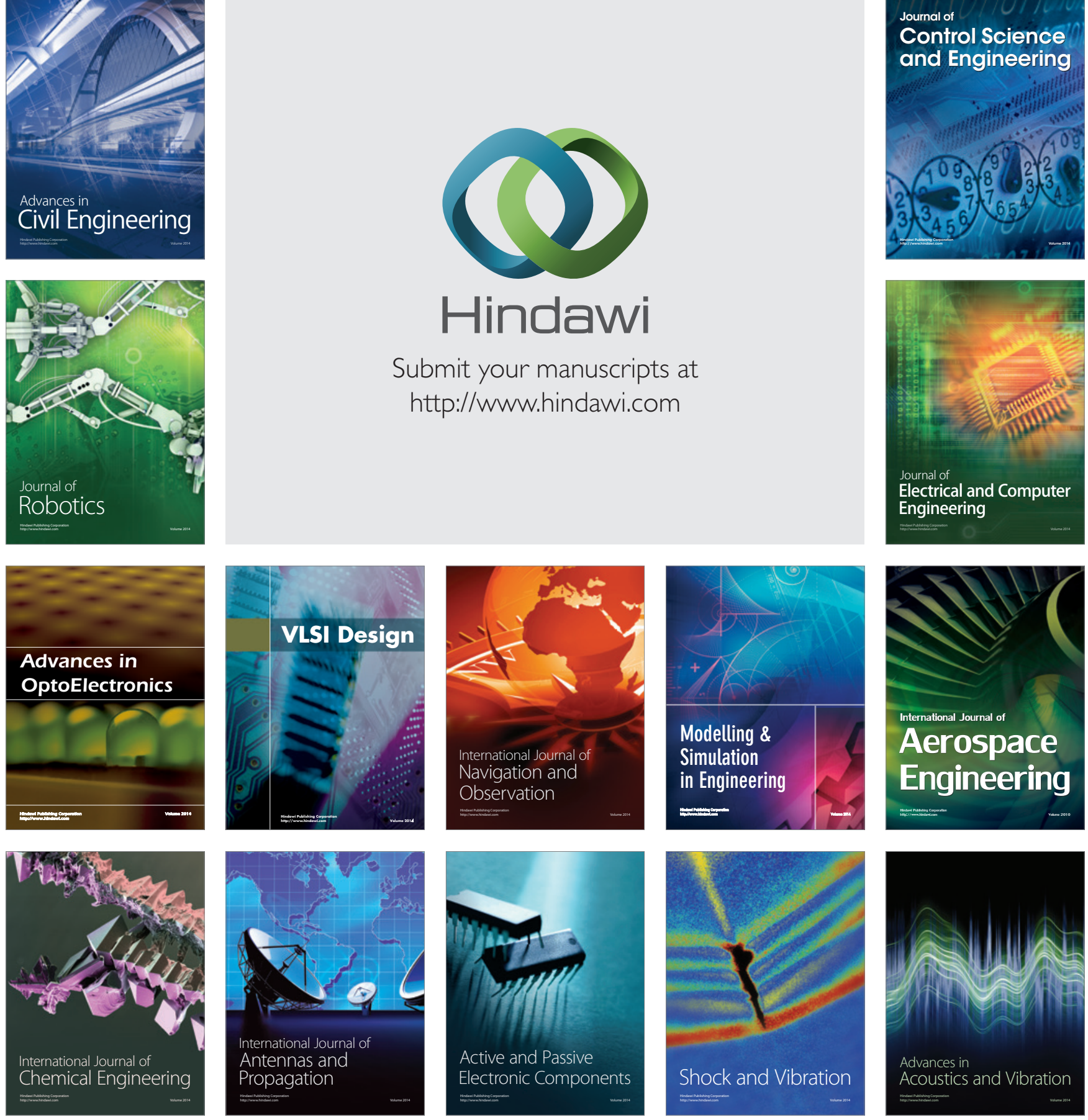